\title{
UN METODO PER LA DETERMINAZIONE DELL'ETA' DELLA TERRA
}

\author{
C. Festa - M. Santangelo
}

La determinazione dell'età della Terra costituisce uno dei problemi, che oggi interessano maggiormente la Geofisica e numerose altre scienze: esso è connesso con quello relativo all'origine del sistema solare $\left(^{1-1}\right)$ e con le altre questioni di carattere ancor più generale, riguardanti l'età dell'universo ${ }^{(5-6)}$, la distribuzione degli elementi nel cosmo $\left({ }^{7-10}\right)$ ed il processo di formazione delle specie nucleari $\left({ }^{11-14}\right)$. Tra i vari metodi escogitati per la determinazione dell'età della Terra $\left({ }^{15}\right)$, quello basato sul fenomeno della radioattività naturale $\left({ }^{16}\right)$ è certamente il più adatto a fornire risultati quantitativi attendilili, in quanto si può assumere con sufficiente correttezza cle le costanti di decadimento degli elementi radioattivi siano rimaste invariate $\left({ }^{17}\right)$ dall'epoca della solidificazione della crosta terrestre: e, generalmente, per età della Terra si intende appunto il tempo trascorso dalla solidificazione della crosta.

Però dalle misure del contenuto degli elementi radioattivi nei singoli campioni si ricava l'età dei minerali $\left({ }^{18}\right)$ : il valore più elevato, ottenuto finora con queste misure, è quello di $2,1 \times 10^{\circ}$ an, relativo ad alcuni campioni del tardo pre-Cambriano, originari del Canadà $\left({ }^{19}\right)$. Questo valore può essere considerato come un limite inferiore dell'età della Terra. Holmes $\left({ }^{20}\right)\left({ }^{22}\right)$, elaborando i risultati delle analisi isotopiche di 25 campioni di minerale di piombo, eseguite da Nier e coll. $\left({ }^{21}\right.$ ), ha ottenuto per l'età della terra il valore $3,35 \times 10^{8}$ an. La sua determinazione ha il vantaggio di hasarsi su misure eseguite con lo spettrografo di massa e quindi molto precise: essa risente però indubluiamente dei criteri statistici con cui vengono elaborati questi dati e principalmente della estrapolazione a tutta la terra di valori limitati ad alcuni campioni.

Per evitare questi inconvenienti ci è sembrato opportuno studiare un metodo, che consenta una valutazione della età della terra, fondata su dati relativi all'intera crosta, anziché a singoli minerali. Evidentemente questa valutazione è possibile solo nel caso in cui si 
disponga di analisi molto accurate e riferite ad un grande numero di campioni provenienti da differenti zone della terra, in maniera da conoscere con buona approssimazione il contenuto medio dell'elemento radioattivo considerato e dei suoi prodotti di decadimento stabili nell'intera crosta. Questa condizione è soddisfatta dal potassio, mentre non lo è dai tre capostipiti delle famiglie radioattive ( ${ }_{92} U^{238}$, ${ }_{02} U^{235}$ e $\left.{ }_{90} T h^{232}\right)$ né dagli altri elementi radioattivi naturali $\left({ }_{37} R b^{87}\right.$, ${ }_{71} L u^{170},{ }_{75} R e^{187},{ }_{62} S a^{148}$ ).

E noto infatti che uno dei principali costituenti della crosta terrestre è il calcio e che il più abbondante fra i suoi isotopi è il ${ }_{00} \mathrm{Ca}^{40}$ $(96,9 \%)$, il quale compare fra i prodotti di disintegrazione del ${ }_{19} K^{40}$, isotopo radioattivo del potassio, altro elemento molto diffuso sulla terra. Il metodo esposto in questa nota sfrutta appunto la disintegrazione del ${ }_{19} \mathrm{~K}^{40}$ in ${ }_{20} \mathrm{Ca}^{40}$, in quanto questi due elementi sono abbastanza diffusi sulla crosta terrestre da consentire una valutazione del loro contenuto medio con una approssimazione sufficiente alla applicazione del metodo stesso.

Il processo di disintegrazione del ${ }_{19} K^{40}$ è stato studiato da vari autori $\left({ }^{23-28}\right)$ : il doppio decadimento è ormai confermato sperimentalmente $\left({ }^{29-35}\right)$. Le più recenti determinazioni della costante di decadimento per emissione $\beta-, \lambda_{\beta}$, e della costante di decadimento totale $\lambda$, forniscono $\left({ }^{36}\right)$ :

$$
\begin{aligned}
& \lambda_{\beta}=0,5 \times 10^{-9} \mathrm{an}^{-1} \\
& \lambda=1,97 \times 10^{-9} \mathrm{an}^{-1}
\end{aligned}
$$

Il numero $N_{c a}$ di atomi di ${ }_{20} \mathrm{Ca}^{40}$, formatisi in un tempo $t$ in conseguenza del decadimento del ${ }_{10} K^{40}$, è dato dalla nota equazione:

$$
N_{\mathbf{c a}}=N_{\mathbf{k}} \frac{\lambda_{\rho}}{\lambda}\left(e^{\lambda t}-1\right)
$$

dove $N_{\mathrm{K}}$ è il numero di atomi di ${ }_{99} K^{40}$ rimasti. Dalla [1] si può ricavare l'età di formazione della crosta terrestre, purché si ammetta che tutto l'isotopo di massa 40 del calcio sia di origine radioattiva - ipotesi che, come vedremo tra poco, è assolutamente inaccettabile - e si conosca il contenuto in calcio e potassio della crosta terrestre insieme con la composizione isotopica dei due elementi. Questi dati si ricavano dalle analisi di Clarke e Washington $\left({ }^{37}\right)$ e dalle tabelle di Seaborg e Perlmann ( $\left.{ }^{38}\right)$. Con essi si ottiene per $t$ un valore di $5,4 \times 10^{9}$ an, che può essere assunto come un limite massimo della età della Terra. 
In conseguenza di ciò e di quanto è stato detto nell'introduzione, l'età della terra sarebhe compresa fra 2,1 e $5,4 \times 10^{\circ}$ an, ossia fra i limiti che molti autori impongono all'età della terra $\left({ }^{39-10}\right)$ e all'età dell'universo $\left({ }^{6}\right)$.

Per ottenere una determinazione più precisa di $t \grave{e}$, naturalmente, indispensabile la conoscenza della quantità di ${ }_{20} \mathrm{Ca}^{40}$, presente all'epoca della formazione della crosta.

Anzitutto si può osservare da un punto di vista qualitativo che il nucleo ${ }_{20} \mathrm{Ca}^{40}$ deve essersi formato inizialmente in grande abbondanza, poiché esso è costituito da 20 neutroni e 20 protoni ed il numero 20 è uno di quei numeri magici ai quali compete una stabilità nucleare particolarmente elevata $\left({ }^{41}\right)$. Conseguenza di ciò è appunto la grande abbondanza di questo elemento nel cosmo $\left({ }^{10}\right)$ e il considerevole numero di isotopi stabili che lo compongono. Osserviamo inoltre che il nucleo ${ }_{20} \mathrm{Ca}^{40}$ appartiene a quella ristretta categoria di nuclei con eccesso neutronico zero e numero massico multiplo di quattro, che sono generalmente molto abbondanti nel cosmo e sono contenuti in alta percentuale nell'elemento a cui appartengono.

TABELLA I

\begin{tabular}{|c|c|c|c|c|}
\hline Elemento & Z & A & $\begin{array}{c}\text { A b boud a nza } \\
\text { isotopica }\left({ }^{98}\right) \\
i_{0}\end{array}$ & $\begin{array}{l}\text { Abbondanza cosmi- } \\
\text { ca }\left({ }^{20}\right) \text { riferita a } 10^{4} \\
\text { atomi di Si }\end{array}$ \\
\hline o & 8 & 16 & 99.7 & 220000 \\
\hline $\mathrm{Ne}$ & 10 & 20 & 90,5 & 42.000 \\
\hline $\mathrm{Mr}_{\mathrm{r}}$ & 12 & 24 & 78.6 & 6.970 \\
\hline $\mathrm{Si}$ & 14 & 28 & 92,3 & 9.228 \\
\hline S & 16 & 32 & 95,1 & 3.300 \\
\hline A & 18 & 36 & 0,31 & 450 \\
\hline $\mathrm{Ca}$ & 20 & 40 & 96,9 & 650 \\
\hline
\end{tabular}

Tali specie nucleari sono riportate nella tabella $I$ : in essa mancano i nuclei leggeri, ${ }_{2} \mathrm{He}^{4}$ e ${ }_{6} \mathrm{C}^{12}$, i cui rapporti di abbondanza isotopica possono ess sre variati rispetto a quelli originari $\left({ }^{8}\right)$, in conseguenza di processi termonucleari tuttora in atto nelle stelle $\left({ }^{42}\right)$.

La sola eccezione della tabella I è costituita dall' ${ }_{18} A^{36}$. Riguardo a questo nucleo si può osservare, però, che, siccome è impossibile ri- 
trovare nelle meteoriti quantità minime di sostanze volatili, quali i gas rari, mentre d'altro canto $i$ dati stellari ad essi relativi sono insufficienti, le loro abbondanze cosmiche sono ancora praticamente sconosciute $\left({ }^{10}\right)$; nel caso particolare $\operatorname{dell}_{18} A^{36}$ bisogna inoltre notare che la sua abbondanza isotopica non è quella originaria in quanto l'isotopo più abbondante, ${ }_{18} A^{40}$, è generato $\left({ }^{43-40}\right)$ sulla terra dal ${ }_{10} K^{40}$.

In base a quanto abbiamo detto sopra possiamo concludere che è da scartare in maniera definitiva l'ipotesi, temporaneamente ammessa, secondo la quale tutto il ${ }_{20} \mathrm{Ca}^{40}$ si è formato in conseguenza del decadimento del ${ }_{10} K^{40}$; si deve anzi ritenere con una certa sicurezza che nel processo generale di formazione delle specie nucleari, il nucleo ${ }_{20} \mathrm{Ca}^{40}$ deve essere stato generato in abbondanza così come gli altri nuclei elencati nella tabella I.

Sorge quindi il problema della determinazione della quantità originaria di ${ }_{20} \mathrm{Ca}^{40}$ dalla quale si possa partire per valutare il rapporto fra ${ }_{20} \mathrm{Ca}^{40}$ radiogenico e ${ }_{10} \mathrm{~K}^{40}$, che risulta funzione del tempo $t$ da calcolare. Osserviamo a questo proposito che esistono numerose analisi di meteoriti dalle quali si può ricavare con discreta precisione $\left({ }^{47}\right.$ ). il contenuto percentuale di calcio e potassio: con opportune ipotesi, la validità delle quali sarà discussa in seguito, si può scrivere un sistema di equazioni dalle quali si ricava il tempo $t$ e le percentuali di ${ }_{20} \mathrm{Ca}^{40}$, contenute originariamente delle meteoriti e nella crosta terrestre.

Indichiamo con $b$ l'attuale contenuto percentuale in peso di ${ }_{20} \mathrm{Ca}^{40}$ nella crosta terrestre, con $x$ il ${ }_{20} \mathrm{Ca}^{40}$ presente nella crosta al tempo della sua formazione e con quello formatosi per decadimento del ${ }_{19} K^{40}$ durante il tempo $t$. Evidentemente si ha:

$$
b=x+\xi
$$

D'altra parte se con $a$ si indica l'abbondanza isotopica percentuale del ${ }_{20} \mathrm{Ca}^{40}$ nel calcio totale al tempo di formazione della crosta, si ha:

$$
x=\alpha X
$$

dove $X$ è la quantità di calcio totale presente nella crosta terrestre al tempo $t$.

Ma se con $a$ si indica la quantità di calcio totale presente attualmente nella crosta terrestre, si può scrivere:

$$
X=a-\xi
$$


dimodoché, introducendo la [4] nella [3] si ha:

$$
x=\alpha(a-\xi)
$$

Se con le stesse lettere contrassegnate da un apice si indicano le corrispondenti quantità per le meteoriti, si ottengono parallelamente alla [2] e alla [5] le due equazioni:

$$
\begin{aligned}
& b^{\prime}=x^{\prime}+\xi^{\prime} \\
& x^{\prime}=\alpha^{\prime}\left(a^{\prime}-\xi^{\prime}\right)
\end{aligned}
$$

Se ammettiamo $-1^{\mathfrak{a}}$ ipotesi - che sia:

$$
\alpha=\alpha^{\prime}
$$

dividendo membro a membro le due equazioni [5] e [5'] si ha:

$$
\frac{x}{x^{\prime}}=\frac{a-\xi}{a^{\prime}-\xi^{\prime}}
$$

si ottiene così in definitiva il sistema di equazioni

$$
\begin{aligned}
& b=x+\xi \\
& b^{\prime}=x^{\prime}+\xi^{\prime} \\
& \frac{x}{x^{\prime}}=\frac{a-\xi}{a^{\prime}-\xi^{\prime}}
\end{aligned}
$$

Per calcolare $\xi$ e $\xi$ si parte dalle note leggi del decadimento radioattivo; indicando con $c$ e $c^{\prime}$ la quantità di ${ }_{19} K^{40}$ presente attualmente nella crosta terrestre e nelle meteoriti, si ha:

$$
\begin{aligned}
& \xi=c \frac{\lambda_{\beta}}{\lambda}\left(e^{\lambda t}-1\right) \\
& \xi^{\prime}=c^{\prime} \frac{\lambda_{\rho}}{\lambda}\left(e^{\lambda t^{\prime}}-1\right)
\end{aligned}
$$

dove $t$ è il tempo trascorso dalla formazione della crosta terrestre e $t^{\prime}$ quello trascorso dalla separazione delle fasi meteoriche.

Se si pone $-2^{\mathrm{a}}$ ipotesi - :

$$
t=t^{\prime}
$$


il sistema di equazioni [7] diventa:

$$
\begin{aligned}
& \boldsymbol{b}=\boldsymbol{x}+\boldsymbol{c} \frac{\lambda_{\beta}}{\lambda}\left(e^{\lambda t}-1\right) \\
& \left.b^{\prime}=x+c{\frac{\lambda_{\underline{\beta}}}{\lambda}}^{\lambda}{ }^{\lambda t}-1\right) \\
& \frac{\boldsymbol{x}}{\boldsymbol{x}^{\prime}}-\frac{\boldsymbol{a}-c \frac{\bar{\lambda}_{\overline{\mathrm{p}}}}{\hat{\lambda}}\left(e^{\lambda t}-1\right)}{a^{\prime}-c^{\prime} \frac{\lambda_{\beta}}{\lambda}\left(e^{\lambda t}-1\right)}
\end{aligned}
$$

Allora introducendo nel sistema i valori percentuali $\left({ }^{10}\right)\left({ }^{37}\right)\left({ }^{38}\right)$ :

$$
\begin{array}{ll}
a=3,65 & a^{\prime}=1,97 \\
b=3,54 & b^{\prime}=1,91 \\
c=31 \times 10^{-5} & c^{\prime}=2,4 \times 10^{-5}
\end{array}
$$

insieme con le costanti di decadimento $\left({ }^{30}\right)$ del ${ }_{10} K^{40}$ :

$$
\lambda=1,97 \times 10^{-9} \mathrm{a}^{-1}, \quad \lambda_{\beta}=0,5 \times 10^{-9} \mathrm{a}^{-1}
$$

e risolvendolo, si ottiene:

$$
t=3,16 \times 10^{9} a
$$

e le percentuali:

$$
\begin{aligned}
& x=3,50 \\
& x^{\prime}=1,90
\end{aligned}
$$

L'espressione di $t$, che si ricava dal sistema [9], è

$$
\boldsymbol{t}=\frac{\log \left(b-\frac{x}{c} \frac{\lambda}{\lambda \boldsymbol{\beta}}+1\right)}{\lambda}
$$

Essa ci fornisce un valore di $t$ fondato essenzialmente sulle due ipotesi precedentemente avanzate.

La prima, $\alpha=\alpha^{\prime}$, ciò̀ uguale abbondanza isotopica originaria sulla terra e sulle meteoriti, risulta confermata sperimentalmente dal fatto che le analisi finora eseguite hanno dimostrato che l'abbondanza isotopica terrestre $\left({ }^{10}\right)$ del ferro, nichel, gallio, ossigeno, silicio, cloro, 
e cobalto è identica a quella meteorica e, poiché si tratta di elementi stabili, è presumibile che la composizione isotopica attuale coincida con quella originaria. Questo è tanto più vero in quanto le piccole variazioni nelle composizioni isotopiche, che qualche volta si riscontrano sulla terra $\left(^{(48-51}\right)$ sono per noi trascurabili, perché $i$ nostri valori rappresentano medie di analisi eseguite su numerosi campioni.

Relativamente alla seconda ipotesi, $t=t^{\prime}$, non esistono sinora prove decisive: si può osservare solamente che le misure di età delle meteoriti effettuate da Paneth e coll. $\left({ }^{52}\right)$, non rivelano alcuna differenza sostanziale fra $i$ valori delle due età, considerate come il tempo trascorso dall'epoca della separazione delle fasi per le meteoriti e rispettivamente per la terra. Si noti a questo proposito che, mentre da un lato sono da scartare i valori più bassi, ottenuti da questi AA., perché il metodo dell'elio dà sistematicamente scarti in tal senso a causa della continua perdita di questo gas nel tempo, d'altro canto anche i valori più elevati sono suscettibili di critica in quanto non è improbabile che nelle meteoriti sia presente un eccesso di elio generato in processi nucleari, originati dall'urto di nucleoni della radiazione cosmica contro la materia meteorica $\left({ }^{53-54}\right)$. In tal modo si potreblbero spiegare i valori più alti ottenuti da Paneth: del resto se si applica alle meteoriti il metodo seguito per trovare il limite massimo della età della terra dal rapporto ${ }_{90} \mathrm{Ca}^{40} /{ }_{19} \mathrm{~K}^{10}$, si trova un valore massimo dell'età delle meteoriti pari a $6,4 \times 10^{0}$ anni, che supera solo del $17 \%$ il valore massimo pertinente alla terra.

Sull'origine delle meteoriti sono state avanzate parecchie ipotesi in questi ultimi anni. Secondo Brown $\left({ }^{55-57}\right.$ ), Suess $\left({ }^{58}\right)$ ed altri $\left({ }^{10}\right)$ esse appartengono al sistema solare: inoltre, Chandrasekhar $\left({ }^{2}\right)$, ter Haar $\left({ }^{3}\right)$, Suess $\left({ }^{59}\right)$ e Ramsey $\left({ }^{60}\right)$ attribuiscono una origine comune a tutti i pianeti del sistema solare, compreso il sole stesso. Se questo modo di vedere fosse corretto, sarebbe confermata la ipotesi seconda. Infine una prova indiretta di quanto è stato detto può riconoscersi nel fatto che le composizioni isotopiche attuali di elementi radioattivi ( ${ }^{(1-62)}$ ) quali l'uranio e il potassio nella terra e sulle meteoriti sono pressoché identiche: ora questo dovrebbe essere vero, partendo dalla $1^{\text {a }}$ ipotesi, $\alpha=\alpha^{\prime}$, indipendentemente dai valori di $t$ e $t^{\prime}$, solo per gli elementi stabili, mentre per elementi instabili, quali l'uranio e il potassio, esso si verifica solo se, insieme con la prima ipotesi, si accetta anche la seconda, $t=t^{\prime}$.

I valori sperimentali introdotti nel sistema [9] sono una riela- 
borazione dei dati di Clarke e Washington $\left({ }^{37}\right)$, Brown $\left({ }^{10}\right)$ e di Seaborg e Perlmann $\left({ }^{38}\right)$.

A nostro avviso, essi sono particolarmente attendiljili in quanto si tratta di analisi di elementi molto diffusi nel cosmo. Per quanto riguarda le analisi chimiche, si può - volendo fare una sia pur grossolana valutazione degli errori - attrilsuire a questi dati uno scarto probalsile di circa il 15\%, di fronte al quale lo scarto delle analisi isotopiche è trascurabile. Aggiungiamo che per le meteoriti sono state prese in considerazione solo le analisi eseguite sulla fase silicea la quale, per i processi di differenziazione subiti, è maggiormente confrontabile con i materiali della crosta terrestre.

In una nota precedente $\left({ }^{30}\right)$ aljljiamo trattato il problema delle costanti di decadimento del ${ }_{19} K^{40}$. Seguendo un metodo, già adottato da Ahrens e Evans $\left({ }^{30}\right)$ abjoiamo calcolato il valore di $\lambda$, basandoci su quello, grià noto con sufficiente approssimazione di $\lambda_{\beta}=(0,5+0,03)$ $\times 10^{-\theta} a^{-1}$. Nel presente lavoro alj]iamo assunto per $\lambda$ il valore di $(1,97-0,4) \times 10^{-9} a^{-1}$, che è la media della nostra determinazione e di quelle riportate nella tabella II del lavoro citato $\left({ }^{36}\right)$.

Introducendo nella [10] gli scarti relativi alle diverse quantità che vi compaiono, si ottiene per l'età della terra il valore di

$$
3,16+0,6 \text { miliardi di anni, }
$$

che è perfettamente coerente con quello calcolato da Holmes $\left({ }^{22}\right)$.

Rom^ - Istituto Nazionale di Geofisica - Aprile 1950.

\section{RIASSUNTO}

Si espongono $i$ risultati di un metodo per la determinazione dell'età della terra basato sul decadimento del potassio. Sfruttando i dati di analisi eseguiti su materiali della crosta terrestre e della fase silicea delle meteoriti e facendo opportune ipotesi, si trova una età di $3,16 \times 10^{9}$ anni con una approssimazione di circa il $19 \%$.

\section{BIBLIOGRAFIA}

(1) JefFneYs H.: The origin of the solar system - M. N. R. A. S., 108, 94, 1948.

(2) Сhandanekhar S.: On a new theory of Weiszäcker on the origin of the solar system - Rev. Mod. Phys. 18, 94, 1946.

(3) TER HAar D.: Studies on the origin of the solur system - Kgl. Danske Vid. Selsk. Mat.-Fys. Medd. 25, n. 3, 1948. 
(4) TER HAAn D.: Recent theories about the origin of the solar system - Science, $107,405,1948$.

(5) KiEnle H.: Der Alter der Sterne und die Expansion der Felt - Naturw, $31,149,1943$.

(6) Tolman R. C.: The age of the universe - Rev. Mod. Phys., 21, 374, 1949.

(7) GoldschmidT V. M.: Geochemische Verteilungsgesetze der Elemente, IX Videnskapsakademien, Oslo, 1938.

(8) Suess H. E.: Uber kosmische Haufigkeit der chemischen Elemente - Zs. Naturf., 2a, 311, 1947.

(9) Suess H. E.: Die kosmische Häufigkeit der chemischen Elemente - Exper., $5,266,1949$.

(10) Brown H.: A table of relative abundances of nuclear species - Rev. Mod. Phys, 21, 625, 1949.

(11) Alpher R. A., Bethe H. and Gamow G. A.: On the origin of chemical elements -- Phys Rev., 73, 803, 1948.

(12) Alpher R. A., Hermann R. and Gamow G. A.: Termonuclear reactions in the expanding universe - Phys. Rev, 74, 1198, 1948.

(13) Alpher R. A.: A neutron-capture theory of the formation and relative abundance of the elements - Phys. Rev., 74, 1577, 1948.

(14) Alpher R. A. and Hermann R. C.: On the relative abundance of the elements - Phys. Rev., 74, 1737, 1948.

(15) Knopf A., Schuchert Ch., Kovarik A. F., Holmes A. and Brown F. W.: The age of the earth - Physics of the earth IV, Wash, 1931.

(16) Holmes A.: Radioactivity and geological time - N. R. C. Bull., 80, 220, 1931.

(17) Festa C. e Santangelo M.: La radioattività della terra - Ann. Geofis., 2, $503,1949$.

(18) Kovanik A. F.: Calculating the age of minerals from radioactivity data and principles - N. R. C. Bull., 80, 72, 1931.

(19) Ahrens L. H.: Geological age: the extreme antiquity of pegmatites from Manitoba - Nature, Lond., 160, 874, 1947.

(20) Holmes A.: An estimate of the age of the earth - Nature, Lond., 157, 680, 1946.

(21) Nier A. O., Thompson R. W. and Murrhex B. F.: The isotopic constitu tion of lead and the measurement of geological time - Phys. Rev, 60, 112, 1941.

(22) Holmes A.: A revised estimate of the age of the earth - Nature, Lond., $159,127,1947$.

(23) v. Weiszacker C.: Uber die Moglichkeit eines duales $\beta$-Zerfalls von Kalium - Phys. Zeits., 38, 623, 1937.

(24) MunlofF W.: Aktivitat von Kalium und Rubidium gemessenen mit dem Elektronenzählrohr - Ann. Physik, 7, 205, 1930.

(25) Thompson F. C. and Rowland S.: Dual decay of potassium - Nature, Lond, 152, 103, 1943.

(26) Bleulen E. und Gabriel M.: Dualer Zerfall des Kalium 40 - Helv. Plys., Acta, 20, 67, 1947.

(27) Grif T.: On the half-life of $K^{40}$. Phys. Rev. 74, 831, 1948.

(28) Bonst L. B. and Floyd J. J.: The radioactive decay of $K^{\prime 0}$ - Phys. Rev., $74,989,1948$. 
(20) Meyer H. A, Schwacheim G. and De Souza Santos M. D.: Decay of K'0 - Phys. Rev, 71, 908, 1947.

(30) Ahreys L. H. and Evans R. D.: The radioactive decay constants of Kio as determined from the accumulation of $\mathrm{Ca}^{\text {in }}$ in ancient minerals - Phys. Rev, 74, 279, 1948.

(31) GRíf T.: On the totale half-lile period of K!0 - Phys. Rev., 74, 1199, 1948.

(32) Suess H. E.: On the radioactivity of K/0 - Phys. Rev., 74, 1209, 1948.

(33) HiRzeL $O$. and WAFfer H.: On the radioactivity of $K^{40}$. Phys. Rev, 74, $1553,1948$.

(34) Stout R. W.: Radioactivity of Kł0 - Phys. Rev., 75, 1107, 1949.

(35) Fineman E. L.: On the decay of $K^{40}$ - Phys. Rev., 75, 1447, 1949.

(36) Festa C. e Santangelo M.: Sul decadimento del Ko - Ann. Geofis., $3,95,1950$.

(37) Clarke F. W. and WaShincton H. S.: The composition of the earth crust U. S. Geol. Survey Prof. Papers, n. 127, 1924.

(38) Seaborc G. T. and PerlmanN I.: Table of isotopes - Rev. Mod. Phys, $20,585,1948$.

(30) JEFFFREYS H.: The earth, its origin, history and physical constitution Cambridge University Press, Lnnd, 1929.

(40) Bnown E. W.: The age of the earth from astronomical data - N. R. C. $80,460,1931$.

(41) Mayer M. G.: On closed shells in nuclei - Phys. Rev, 74, 235, 1948.

(42) Chandrasekhar S.: An introduction to the study of stellar structure . University of Chicago Press, Chicago, 1939.

(43) Harteck P. und Suess H.: Der Argongehalt Kalihaltiger Minerale und die Frage des dualen Zerfalls von $K^{40}$. Naturw, 34, 212, 1947.

(44) Aldrich L. T. and Nier A. O.: Argon 40 in potassium minerals - Phys. Rev., 74, 876, 1948.

(45) Suess H. E.: Die Häufigkeit der Edelgase auf der Erde und im Kosmos . J. Geol., 57, 600, 1949.

(46) Brown H.: The atmosphere of the earth and planets - G. Kuiper, ed, University of Chicago Press, Chicago, 1948, p. 260.

(47) Brown H. and Patterson C.: The composition of meteoritic matter, $I$. J. Geol., 55, 405, 1947.

(48) Thode M. G, Mac Namara J, Lossing F. P. and Collins C. B.: Natural variations in the isotopic content of boron and its chemical atomic weight - J. Am. Chem. Soc, 70, 3008, 1948.

(40) Rankama K.: Note on original isotopic composition of terrestrial carbon J. Geol, 56, 199, 1948.

(50) UREY H. C.: Oxygen isotopes in nature and in the laboratory . Science, $108,489,1948$.

(51) Thode M. G., Mac-Namara J. and CollrNs C. B.: Natural variations in the isotopic content of sulphur and their significance - Can. J. Res. B, 27, 361, 1949.

(52) Arrol W. J, Jaconi B. B. and Paneth F.: Meteorites and the age of the solar system - Nature, Lond, 149, 235, 1942.

(53) BAUER C. A.: Absorption of cosmic rays in meteorites - Phys. Rev, 74, $225,1948$. 
(54) BAUER C. A.: Rate of production of helium in meteorites by cosmic ray's Phys. Rev,. 74, 501, 1948.

(55) Brown H. and Patrerson C.: The composition of meteoritic matter, 111 J. Geol., 56, 85, 1948.

(50) BROWN H.: Meteorites, relative abundances and planets structures - Scient. Monthly, 67, 383, 1948.

(57) Brown H.: The composition of meteoritic matter and the origin of meteorites - Science, 109, 251, 1949.

(58) Suess H. E.: Die Radioaktivitat des Kaliums als mittel zur Bestimmung des relativen Alters der Elemente in Meteorites - Naturw, 27, 702, 1939.

(50) Suess H. E.: Zur Chemie der Planeten- und Meteoritenbildung - Zs. Elektroch, 53, 237, 1949.

(60) Ramsey W. H.: On the constitution of terrestrial planets - M. N. R. A. S, $108,406,1948$.

(61) Schumb W. C, Evans R. D. and Hastings J. L.: Determination of Pa in siliceous, terrestrial and meteoritic material - J. An. Chem. Soc., 61, 3451, 1939.

(62) Schumb W. C., Evans R. D. and Leaders W. M.: Radioactive determination of relative abundance of the isotope $K^{10}$ in terrestrial and meteoritic $K \cdot \mathrm{J}$. Am. Chem. Soc., 63, 1203, 1941. 